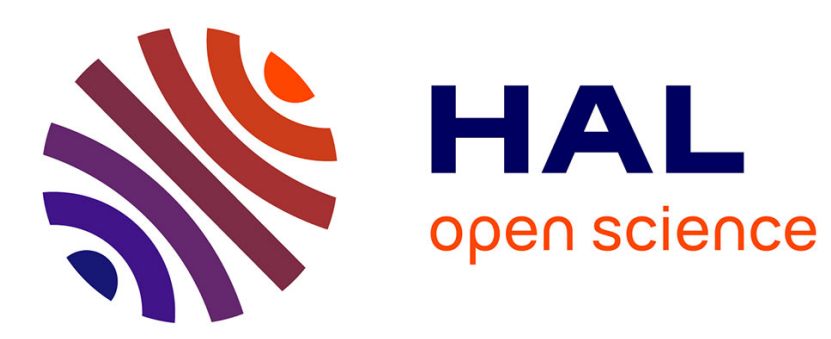

\title{
Mesoscale simulation of aggregation of imogolite nanotubes from potential of mean force interactions
}

Hejian Zhu, Andrew Whittle, Roland J.-M. Pellenq, Katerina Ioannidou

\section{To cite this version:}

Hejian Zhu, Andrew Whittle, Roland J.-M. Pellenq, Katerina Ioannidou. Mesoscale simulation of aggregation of imogolite nanotubes from potential of mean force interactions. Molecular Physics, 2019, 117 (22), pp.3445-3455. 10.1080/00268976.2019.1660817 . hal-02301591

\section{HAL Id: hal-02301591 \\ https://hal.science/hal-02301591}

Submitted on 27 Jan 2020

HAL is a multi-disciplinary open access archive for the deposit and dissemination of scientific research documents, whether they are published or not. The documents may come from teaching and research institutions in France or abroad, or from public or private research centers.
L'archive ouverte pluridisciplinaire HAL, est destinée au dépôt et à la diffusion de documents scientifiques de niveau recherche, publiés ou non, émanant des établissements d'enseignement et de recherche français ou étrangers, des laboratoires publics ou privés. 


\title{
Mesoscale simulation of aggregation of imogolite nanotubes from potential of mean force interactions
}

\author{
Hejian Zhu ${ }^{a}$, Andrew J. Whittle ${ }^{a}$, Roland J.-M. Pellenq ${ }^{a, b}$ and Katerina loannidou ${ }^{a, b, c}$ \\ ${ }^{a}$ Department of Civil and Environmental Engineering, Massachusetts Institute of Technology, Cambridge, MA, USA; ${ }^{\text {b }}<$ MSE $>2$, IRL 3466 \\ CNRS/MIT/Aix-Marseille Université, MIT, Cambridge, MA, USA; 'Laboratoire de Mécanique et Génie Civil, CNRS-Université de Montpellier, \\ Montpellier, France
}

\begin{abstract}
The aggregation of colloidal clay mineral particles plays an important role in controlling the mechan-ical and transport properties of soils. Interactions and aggregation of plate-like montmorillonite particles were previously studied with the help of Molecular Dynamics (MD) simulation. This paper investigates the aggregation of cylindrical imogolite-like phyllosilicate nanotubes. Nano-scale MD simulations are carried out to find the potential of mean force between two nanotubes. This PMF is then used in a mesoscale simulation that represents interactions between elemental nanotubes through coarse-graining. We investigate the distribution of water molecules around the curved surfaces, and the effects of the surface charge density and tube length on aggregation. Shorter nanotubes were found to form larger stacks.
\end{abstract}

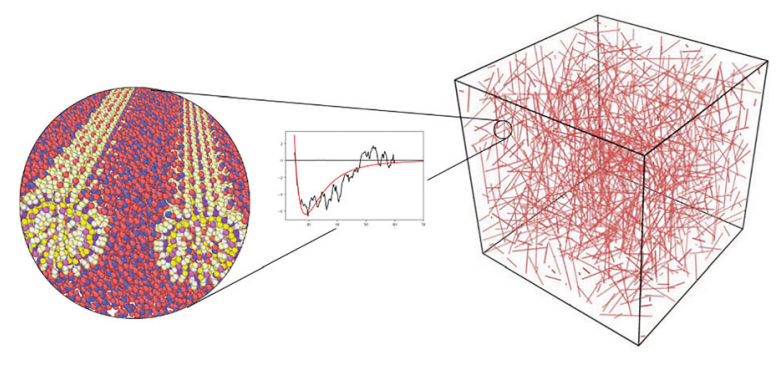

\section{Introduction}

Clay are among the most abundant minerals on the earth with important industrial applications in construction, environmental, pharmaceutical, and process industries. As a particulate material, the aggregation of particles is one of the most important factors governing the bulk properties of clay, including stiffness, strength, and plasticity in the field of mechanical and civil engineering.

Clay minerals have layered structures at nanoscale, most commonly in the form of some combination of tetrahedral silicate and octahedral alumina layers bonded through sharing of oxygen atoms. The layers constructed from the building blocks come in different sizes and can also foil up to form rolls or tubes. Natural imogolite nanotubes are formed as weathering products of volcanic ash and are tubular in shape with a typical diameter $\sim 2 \mathrm{~nm}$, and length $\sim 100$ to $400 \mathrm{~nm}$ [1] (dimension ratio $D / L \approx 1: 50$ to $1: 400$ ). A nanotube consists of an octahedral outer layer and a tetrahedral inner layer, resulting in a thickness of around $5 \AA$ and a chemical formula: $(\mathrm{OH})_{3} \mathrm{Al}_{2} \mathrm{O}_{3} \mathrm{SiOH}$. There also exist double-walled varieties, and types with impurities in the form of isomorphous substitutions (IS) [1]. Through doping, one can also control the diameter of the tubes, which makes imogolite a promising candidate material as molecular sieve [2,3]. Imogolite charged in this way can have a convenient length down to $\sim 10 \mathrm{~nm}$.

Molecular dynamics (MD) method is a powerful technique for studying the interaction between colloidal nanoplatelets at the atomistic scale and can be used to investigate micro-scale aggregation mechanisms through coarse-graining techniques [4]. Various

CONTACT Roland J.-M. Pellenq. pellenq@mit.edu Department of Civil and Environmental Engineering, Massachusetts Institute of Technology, Cambridge, 02139 MA, USA <MSE> 2 , IRL 3466 CNRS/MIT/Aix-Marseille Université, MIT, Cambridge, 02139 MA, USA 
researchers have studied the interactions between platelike particles through molecular simulation, either focusing on the behaviour under different thermodynamic conditions or as a bridging stage between atomistic and mesoscale simulations [4-9]. However, the interactions of particles with curved surfaces have not been investigated in detail. This paper studies the aggregation of imogolite-like aluminosilicate tubular particles at mesoscale through molecular dynamics (MD) simulation. Similar methods have been applied to $\mathrm{Na}-$ montmorillonite in [4] and have provided useful information about the aggregation behaviour, mechanical and transport properties.

In order to get an accurate representation of interparticle interaction, we proceed with free energy perturbation calculations of the water-mediated interaction of two nanotubes, from which the potential of mean force (PMF) (free energy related to the distance between particles) is obtained. The free energy curve (obtained from the atomistic simulations) is then used to calibrate an effective mesoscale pair potential. With the calibrated potential, mesoscale simulations are carried out with coarse-grained particles. We study the geometric properties during the aggregation process for systems with particles of different lengths.

\section{Nanoscale simulation}

\subsection{Simulation methods}

We initially compute the free energy associated with the interactions of two imogolite nanotubes in a parallel configuration. We consider an elemental imogolite using data provided in [10] from X-ray diffraction experiments. The primitive cell $\left((\mathrm{OH})_{3} \mathrm{Al}_{2} \mathrm{O}_{3} \mathrm{SiOH}\right)$ has a height of $8.4 \AA$ containing $1 / 20$ sector of a full cylindrical segment. We construct the typical particle segment containing three repetitive segments resulting in a height of $25.2 \AA$ (Figure 1). In order to simulate the interaction of two tubes, we created an orthorhombic simulation box with dimensions $127.0 \AA(x) \times 63.0 \AA(y) \times 25.2 \AA$ $(z)$, and periodic boundary conditions at all faces. Two segments of tubes were put at a certain separation $d$ in $\mathrm{x}$-direction in the box parallel to each other and to the $z$ axis, aligned along the centre line in the $x$-direction and centered within the unit cell. The centre-to-centre distance between the tubes, denoted by $d$ in the following discussions, will be the controlled parameter, while periodic boundary conditions represent very long imogolite tubes. Horizontal dimensions ( $x$ and $y$ ) of the box are chosen so as to leave enough space from the tube to the boundaries (more than $20 \AA$ clearance) in order to eliminate the effect from images of the tubes in neighbouring periodic copies of the simulation box. In terms of the size of a water molecule $(\approx 3 \AA), 20 \AA$ corresponded to more than 6 layers of water when solvated, which was deemed adequate to eliminate unwanted effects from boundaries or neighbouring images. The height of the cell in the $z$-direction, $25.2 \AA$, corresponds to 3 times the length of the primitive cell of imogolite. After we set the tubes at different spacing $d_{i}$ in place, water molecules are added through a predefined orthogonal lattice of $3.1 \AA$ $(x) \times 3.03 \AA(y) \times 3.2 \AA(z)$, resulting in water density of $0.996 \mathrm{~g} / \mathrm{cm}^{3}$, close to the water density under standard

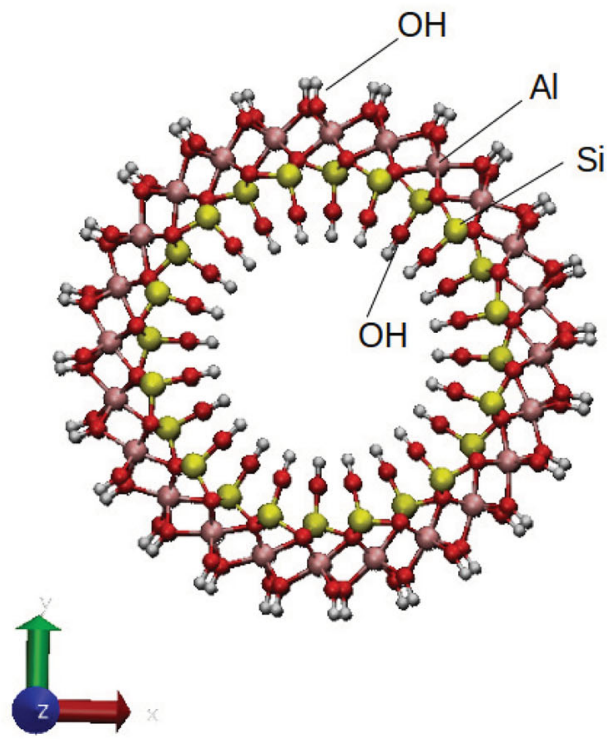

(a)

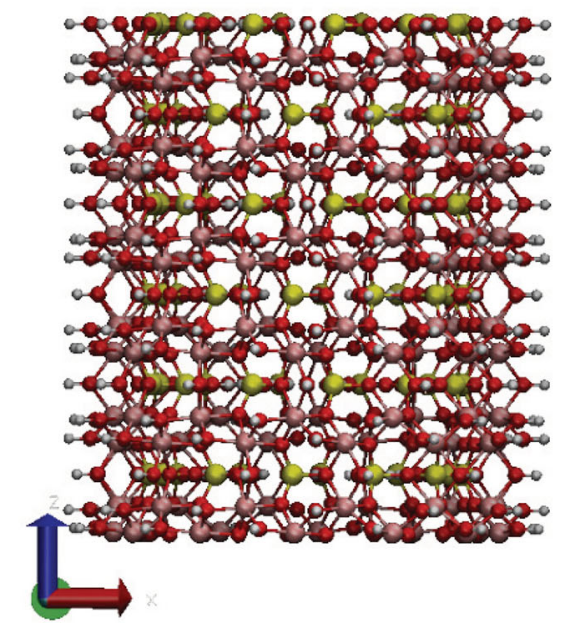

(b)

Figure 1. Representation of a section of the nanotube in the simulation. (a) Plan view and (b) front view. 


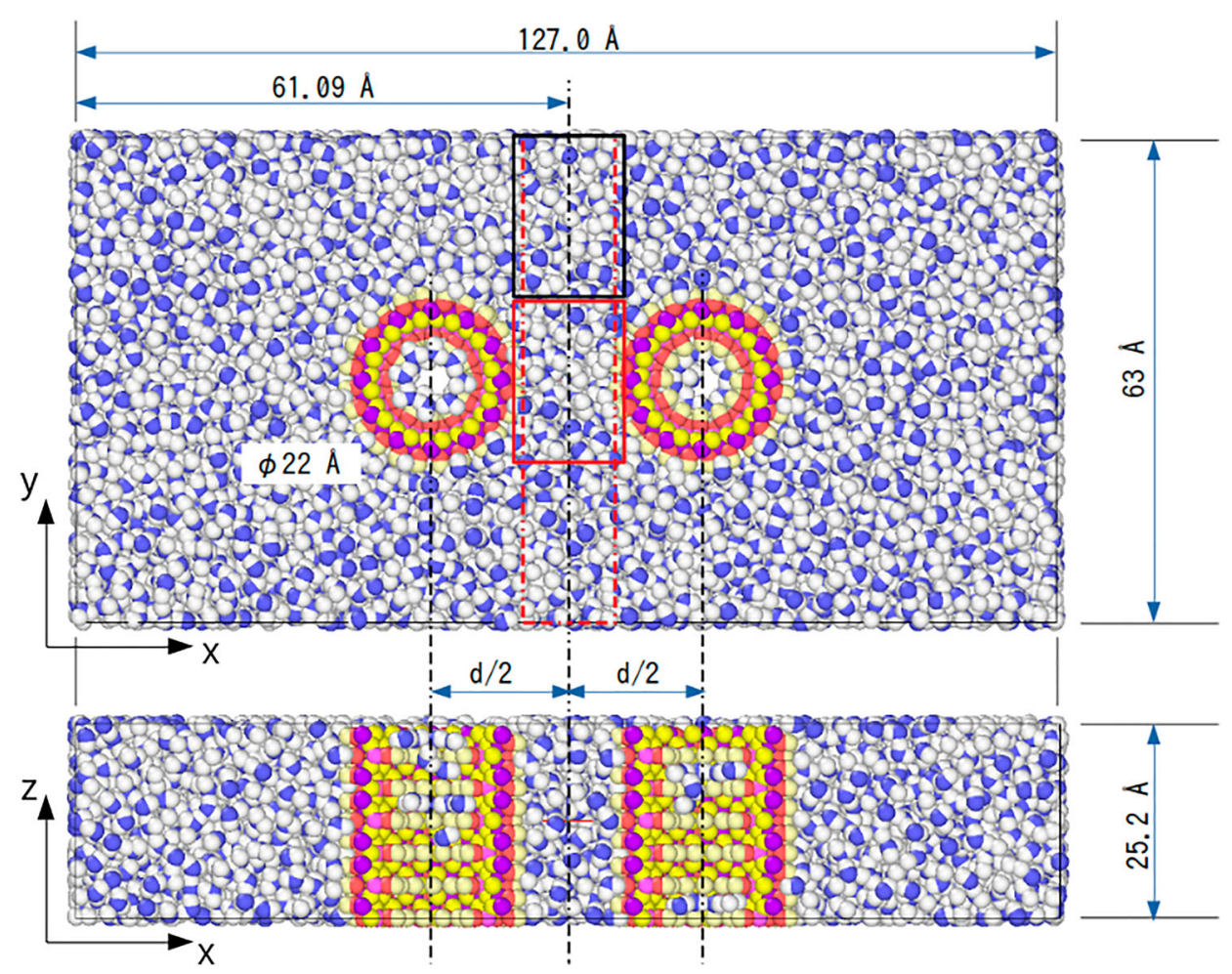

Figure 2. Simulation setup: plan (upper) and front section (lower) views. The configuration was taken after $2.5 \mathrm{~ns}$ NVT simulation of the system with charge-neutral nanotubes. Boxes in the plan view are explained in Sections 3.2 and 3.3.

condition (i.e. $1 \mathrm{~atm}, 298 \mathrm{~K}$ ) [11]. The choice of the lattice constants was to avoid conflict between water molecules from the adjacent periodic simulation box upon creation. Figure 2 shows a typical configuration.

After initial setup, the system was let to evolve in a canonical ensemble (NVT) under constant temperature $300 \mathrm{~K}$ with fixed volume using Molecular Dynamic (MD) simulation with LAMMPS [12]. Temperature control was achieved through the Nosé-Hoover thermostat $[13,14]$. Interactions between atoms were defined by the CLAYFF force field [15], which has been previously used for molecular simulations of clay minerals $[7,16]$. In this model, the potential energy contains four parts: harmonic bond and angle terms for bonded atoms, and van der Waals term of the Lennard-Jones (LJ) type and long-range Coulombic electrostatic term for nonbonded atoms (see Appendix 1). Short-range (LJ) terms were calculated with a potential cut-off of $8.5 \AA$; while long-range Coulombic terms were calculated with the particle-particle-particle-mesh (PPPM) Ewald summation method $[17,18]$. Timestep was set to $1 \mathrm{fs}\left(10^{-15} \mathrm{~s}\right)$. The system was allowed to relax for $500 \mathrm{ps}$ followed by a 2500 ps production period. We recorded 501 configuration, at time intervals of $5 \mathrm{ps}$, during the production stage for each spacing $d_{i}$, for the calculation of the potential of mean force. In the NVT simulations, the imogolite tubes were held fixed and rigid. Bond length and angle of water molecules were constrained with the SHAKE algorithm [19]. The centre-to-centre distance $d_{i}$ ranged from 25 to $60 \AA$, with $0.25 \AA$ increment. The potential of mean force (PMF), corresponding to the Gibbs free energy, between the two tubes was calculated through a free energy perturbation. At each separation, the configurations were perturbed towards $d_{i}+0.25 \AA=d_{i+1}$ and to $d_{i}-0.25 \AA$ $=d_{i-1}$. Differences in potential energy of the perturbed and original states were calculated. The free energy differences between adjacent states were calculated through the following equations [20-22]:

$$
\Delta G\left(d_{i} \rightarrow d_{i \pm 1}\right)=-\frac{1}{\beta} \ln \left[\frac{\langle\exp (-\beta \Delta U / 2)\rangle_{i}}{\langle\exp (-\beta \Delta U / 2)\rangle_{i \pm 1}}\right]
$$

where $d_{i}$ is the starting state, $d_{i \pm 1}$ is the perturbed state, $\beta=1 / k_{B} T$ ( $k_{B}$ is the Boltzmann constant and $T$ is the temperature), and $\Delta U=U\left(d_{i \pm 1}\right)-U\left(d_{i}\right)$. The average was taken over the 501 configurations mentioned above.

The PMF at $60 \AA$ was set to zero as a reference point, and the PMF at other spacings $d_{i}$ was back-calculated through the series of $\Delta G_{i, i+1}$.

To study the effect of surface charge of the nanotubes, we also carried out free energy calculations with charged nanotubes. Natural and synthetic imogolite exhibit relatively high cation exchange capacity [23]. This is mainly due to the surface charge distribution of 
the tubes and from the net charge caused by isomorphous substitution, according to, e.g. Gustafsson [24] and Heckman and Rasmussen [23], in either octahedral or tetrahedral layers. Considering the result by Gustafsson [24], which shows a weak positive local charge in the outer layer and a stronger local negative charge in the inner layer, the surface charge in our simulation was introduced as a result of isomorphous substitution in the tetrahedral inner layer. One Si atom in each $8.4 \AA$ segment was substituted with $\mathrm{Al}$ atom resulting in a configuration with $\mathrm{Si}: \mathrm{Al}=294: 138=2.13$, leaving a net surface charge of around $0.0276 \mathrm{C} / \mathrm{m}^{2}$. To eliminate asymmetry, substitutions in consecutive segments are $120^{\circ}$ from each other in the plan view. To keep the system charge neutral, $\mathrm{Na}^{+}$ ions were added as counterions. Since the system has infinitely long nanotubes, which does not allow material exchange between spaces inside and outside the tubes, we considered two charged cases of which one has counterions within the tubes (case A for brevity) and the other outside the tubes (case B).

\subsection{Potential of mean force}

PMF curves for the charge-neutral case through averaging over different lengths of MD trajectories are shown in Figure 3. The calculated free energy exhibits good convergence after averaging over $2.5 \mathrm{~ns}$. In Figure 3(b), all PMF curves are obtained through averaging over $2.5 \mathrm{~ns}$. The PMF curves exhibit characteristic oscillatory features on top of a general trend, with approximately equally spaced local minima by the size of a water molecule $(3 \AA$, as is shown by the arrows on the bottom curve as an example). The interaction is repulsive when two tubes are close to each other. In the charge-neutral case and case $\mathrm{B}$, there are no dominant potential wells, which is different from that of platelike particles, as presented in [7] in the case of Na-montmorillonite and [8] in the case of graphene, where there are much smaller oscillations in PMF. In order to understand the differences between platelike and cylindrical particles or more generally between slit pores and open-pore space with curved surfaces, we also studied the water distribution in the next section.

PMFs for both uncharged case and case B indicate mostly repulsion between particles. We have found in case A the charged nanotubes together with the counterions inside the tube exhibit net electric dipole moment in the direction aligned with the tubes. The dipole moments are largely constant during each NVT simulation. When the distance between the tubes is small, the two dipole moments tend to be in the same direction. When the distance between the tubes is greater than $30 \AA$ (corresponding to the minimum of the potential well), they tend to

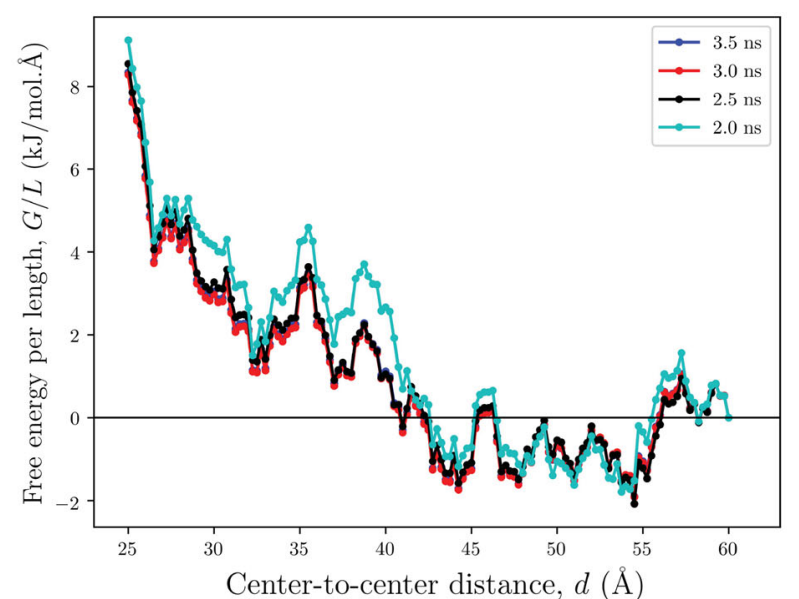

(a)

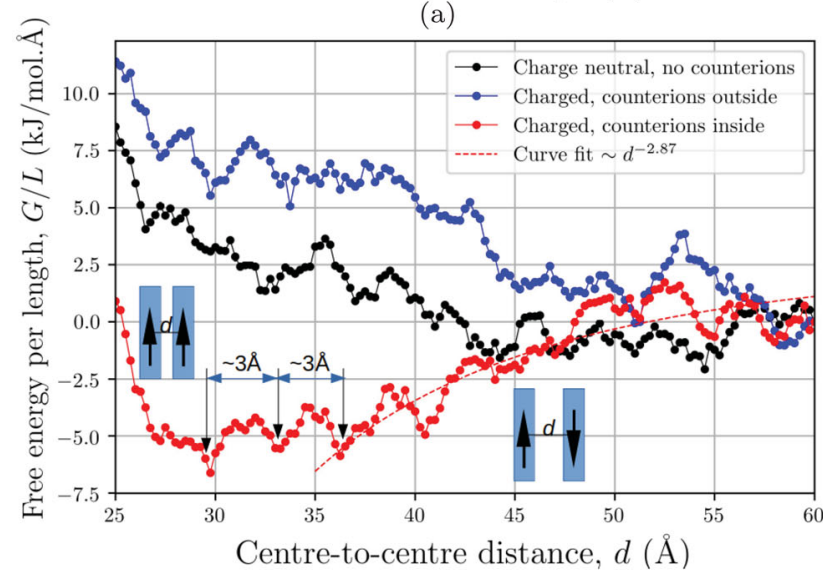

(b)

Figure 3. (a) Convergence of potential of mean force curve for charge-neutral case; (b) potential of mean force curve for cases with charge-neutral particles (middle), charged particles with counterions outside (upper) and inside (lower). The horizontal axis is the distance between the two nanotubes, and the vertical axis the free energy or potential of mean force per unit length in the longitudinal direction.

be in the opposite direction. The magnitude of the dipole moments is largely the same. As a first approximation, we also did a power law fitting for the tail part ( $d$ greater than $35 \AA$ ) of the PMF curve for Case A and obtained a best fit power exponent of -2.87 (with correlation coefficient 0.76 ), which is quite close to dipole-dipole interaction's linear dependence upon $d^{-3}$. Since our aim is to investigate microstructures that develop due to aggregation, we have focused our subsequent mesoscale simulations on charged imogolite nanotubes with interior counterions (Case A) only.

\subsection{Kernel density distribution of water molecules and hydrogen bonds}

Kernel density estimation was carried out for oxygen atoms in water molecules and hydrogen bonds. It is 


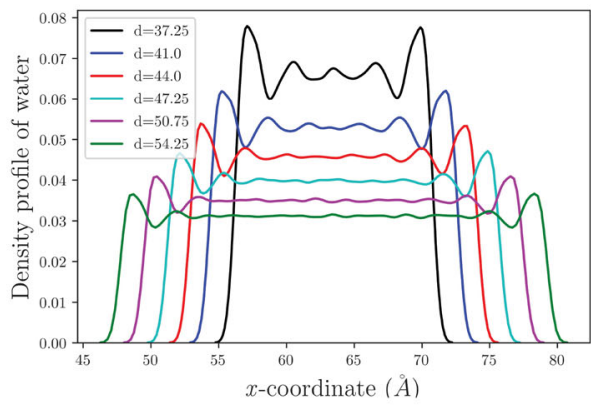

(a)

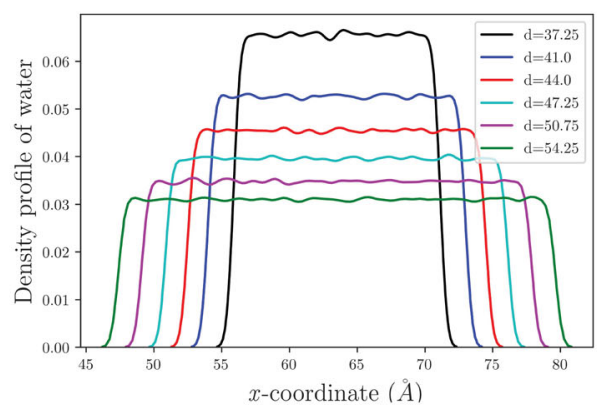

(c)

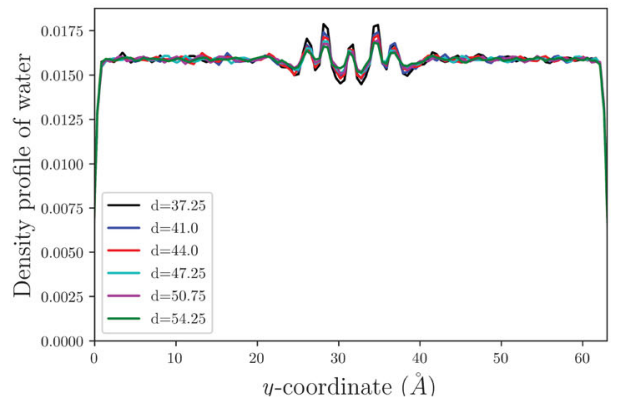

(e)

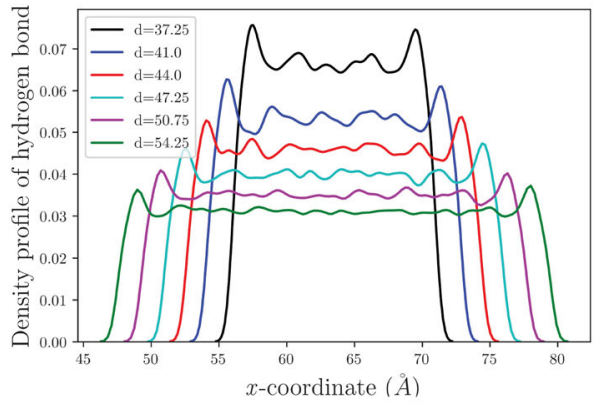

(b)

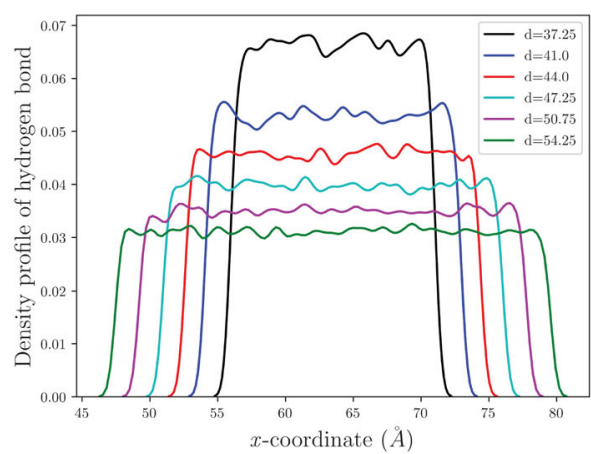

(d)

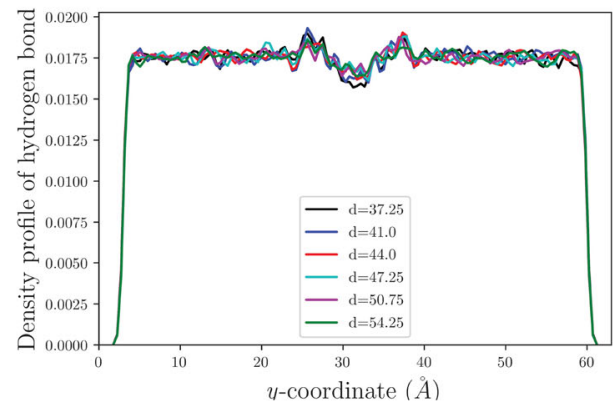

(f)

Figure 4. Probability density distribution of water molecules $(a, c, e)$ and hydrogen bonds $(b, d, f)$. The plots were produced with kernel density estimation averaged over 500 configurations. Vertical axes are the likelihood to find an oxygen atom of water/a hydrogen bond. (a) Water, between (lower solid box), (b) hydrogen bonds, between (lower solid box), (c) water, beside (upper solid box), (d) hydrogen bonds, beside (upper solid box), (e) water, across, and (f) hydrogen bonds, across (dashed box).

equivalent to a smoothed histogram or a probability density function. The vertical axes of these plots indicate the relative likelihood of finding an oxygen atom or hydrogen bond. In the following discussion, the name 'density distribution' is used for short. Each curve is the result of averaging over 500 configurations over $2.5 \mathrm{~ns}$. Figure 4(a) shows the density distribution along the $x$-axis of oxygen atoms in water molecules within the region between the tubes indicated by the lower solid box in Figure 2. For a certain $d_{i}$, the region is $x \in\left[63.5-\left(d_{i} / 2\right)+11.09,63.5+\right.$ $\left.\left(d_{i} / 2\right)-11.09\right]=\left[74.59-\left(d_{i} / 2\right), 52.41+\left(d_{i} / 2\right)\right]$ and $y \in[31.5-11.09,31.5+11.09]=[20.41,42.59]$. In Figure 4(a), the density profiles share similar distributions, with the highest density close to the surface of the nanotubes. This corresponds to a formation of a denser water layer in the vicinity of the tubes comprising $1-2$ layers of water molecules.

Structuring of water molecules is more prominent in the region between the tubes. In Figure 4(c), distribution of water molecules along $x$-direction within the black box in Figure 2 is shown. For certain $d_{i}$, the region is $x \in\left[74.59-\left(d_{i} / 2\right), 52.41+\left(d_{i} / 2\right)\right]$ and $y \in[31.5+$ $11.09,63.0]=[42.59,63.0]$. Unlike in Figure 4(a), the peaks are less distinguishable in the density distribution plots.

Along the perpendicular bisector of the centre-tocentre line segment, we could also observe geometric features of water molecules. Figure 4(e) shows the distribution of water molecules along the $y$-direction within the dashed box shown in Figure 2, corresponding to the region: $x \in\left[74.59-\left(d_{i} / 2\right), 52.41+\left(d_{i} / 2\right)\right]$. Beyond 
the edges of the two tubes (that is, $y \in[0,20.41) \cup$ $(42.59,63.0])$, water molecules are less structured. Within the region covered by the lower solid box (that is, $y \in[20.41,42.59])$, distribution curves show peaks at around fixed positions. The peaks, however, are not equidistant from adjacent ones and are not of equal magnitude, but are symmetric with respect to the centre line.

We also investigated the distribution of hydrogen bonds within the above-mentioned regions. A commonly used geometric definition was adopted: a hydrogen bond is said to be established when $\mathrm{O}-\mathrm{O}$ distance of two water molecules is less than $3.5 \AA$, and that the $\mathrm{H}-\mathrm{O}-\mathrm{O}$ angle is less than $30^{\circ}$ [25]. As shown in Figure 4(b,d,f), hydrogen bonds were found to have similar distribution corresponding to that of bulk water.

\section{Mesoscale simulation}

\subsection{Coarse-graining technique}

Mesoscale simulations were carried out using the PMF obtained in the nanoscale simulation. Nanotubes were coarse-grained into a series of spherical subparticles (Figure 5) treated as rigid bodies. Subparticles are linearly aligned. Interaction between two nanotubes was treated as the sum of pair-wise interactions between subparticles, with intra-tube interactions excluded, i.e. the potential energy of tubes $A$ and $B$ is,

$$
U_{A B}=\sum_{\alpha \in A} \sum_{\beta \in B} u_{\alpha \beta}
$$

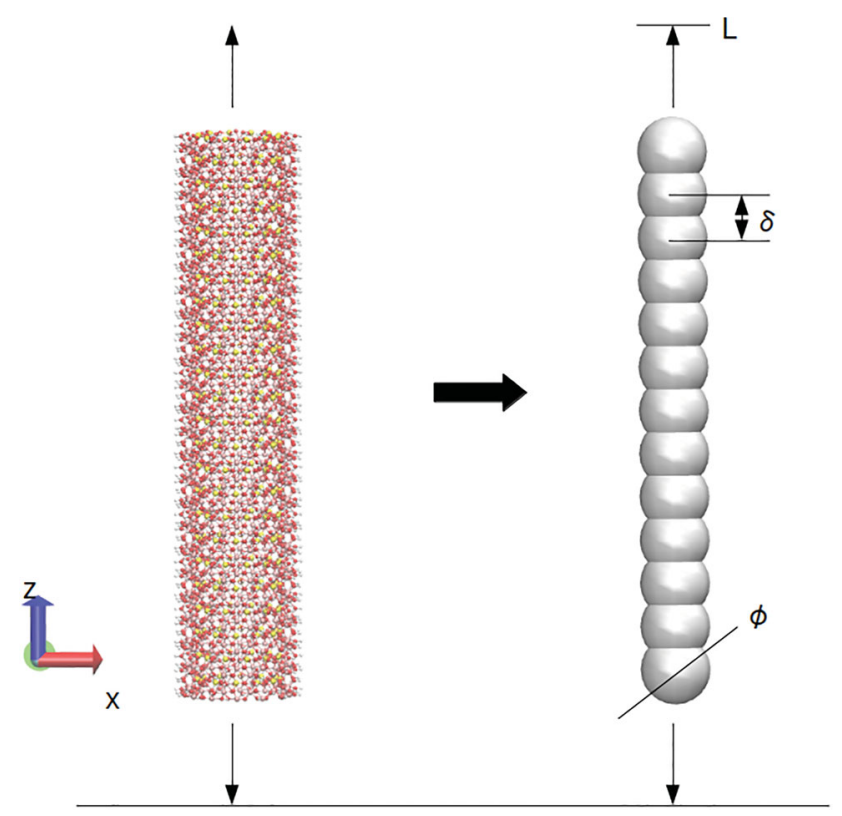

Figure 5. Schematic representation of the coarse-grained particle, rendered with Visual Molecular Dynamics (VMD) [26].

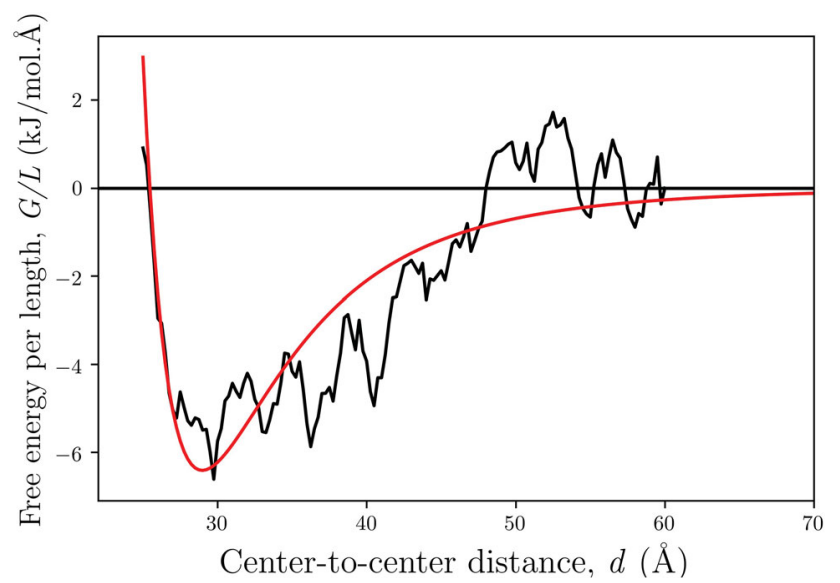

Figure 6. Curve-fitting with $L J$ and $L J / C o u l o m b$ potential forms according to free energy per length data.

Table 1. Parameters for systems with coarse-grained tubes of different lengths.

\begin{tabular}{lccccc}
\hline Name for the system & CG-12 & CG-20 & CG-24 & CG-30 & CG-40 \\
\hline Number of sub-particles & 12 & 20 & 24 & 30 & 40 \\
Equivalent tube length (A) & 100.8 & 168.0 & 201.6 & 252.0 & 336.0 \\
Number of tubes & 2000 & 1200 & 1000 & 800 & 600 \\
Total simulation time (ps) & 5000 & 5500 & 6500 & 7500 & 8500 \\
\hline
\end{tabular}

LJ potential is adopted as a first approximation, that is, the energy between subparticles $\alpha$ and $\beta$ is

$$
u_{\alpha \beta}^{L J}=4 \epsilon\left[\left(\frac{\sigma}{r_{\alpha \beta}}\right)^{12}-\left(\frac{\sigma}{r_{\alpha \beta}}\right)^{6}\right]
$$

The parameters were obtained through curve fitting in terms of free energy per length (Figure 6), which provides a good approximation of the trend in the computed PMF. We considered tube lengths ranging from 100.8 to 336 angstroms, equivalent to radius to length ratios of 1:10 to $1: 30$ (Table 1 and Section 3.2), so that it is safe to assume curve-fitting according to results from simulations with two tubes in parallel can provide us with satisfactory results.

The fitted values are shown in Table 2 together with other parameters. Mass and radius of subparticles were chosen so at to represent the mass and longitudinal moment of inertia of the corresponding segment of imogolite.

\subsection{Simulation setup}

The mesoscale simulation was implemented using LAMMPS [12]. We start with configurations with 32 tubes within a cubic simulation box with side length $100 \mathrm{~nm}$ on a cubic lattice $(a=500 \AA)$, followed by a grand-canonical Monte Carlo (GCMC) simulation to 
Table 2. Parameters for mesoscale force field.

\begin{tabular}{lcc}
\hline Parameter & Short & Unit \\
\hline$\epsilon$ & 2.294 & $\mathrm{kcal} / \mathrm{mol}$ \\
$\sigma$ & 9.6 & $\mathrm{~kJ} / \mathrm{mol}$ \\
$\AA$ & 27.1 & $\AA$ \\
Subparticle mass & 4752.69 & $\mathrm{~g} / \mathrm{mol}$ \\
Subparticle diameter, $\phi$ & 27.27 & $\AA$ \\
Subparticle spacing, $\delta$ & $8.4^{*}$ & $\AA$ \\
\hline
\end{tabular}

Notes: Calculation of mass and diameter of subparticles is shown in the appendix. $\left(^{*}\right)$ subparticle spacing is actually smaller than the radius: subparticles were overlapping. But since particles were treated as rigid bodies, this would not generate problem or unphysical phenomena.

put in enough nanotubes without overlapping. We considered five monodispersed cases. The total number of subparticles was kept constant to be 24,000, and the lengths of the tubes were chosen to be 100.8 , $168,201.6,252$, and $336 \AA$, corresponding to 12,20 , 24,30 , and 40 subparticles per tube, respectively. In the following discussion, the systems will be named 'CG' (representing 'coarse-grained') followed by the tube size. The systems were then relaxed with MD simulation in a canonical ensemble (NVT) at $300 \mathrm{~K}$ for more than $5.0 \mathrm{~ns}$ with a time step of $1 \mathrm{fs}$. The duration of their respective simulations is sufficient to allow full relaxation of the system (see Section 3.4). Temperature control was achieved with the Nosé-Hoover thermostat $[13,14]$.

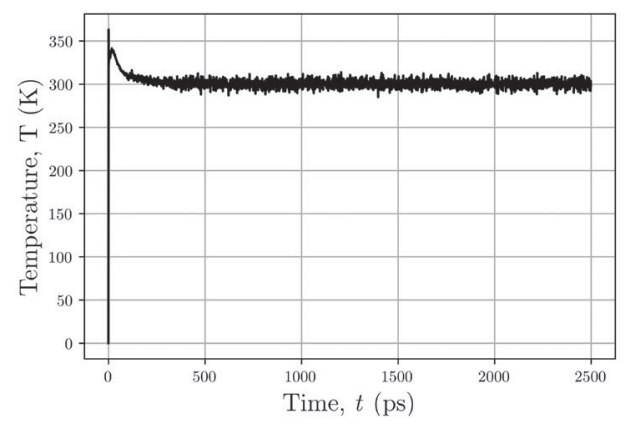

(a) temperature

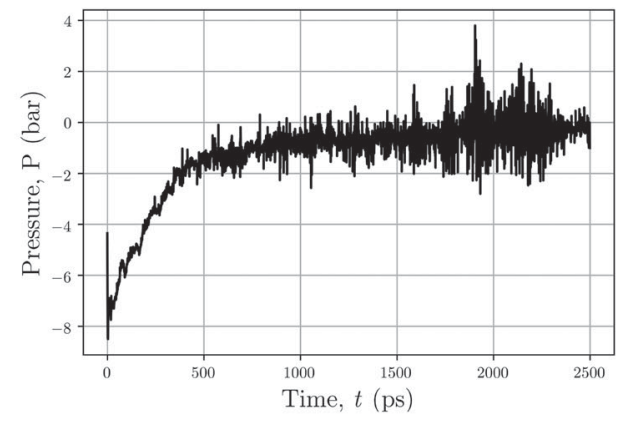

(b) pressure

\subsection{Validation of simulation}

\subsection{Aggregation behaviour}

To check the convergence of the systems, we examined the thermodynamic variables. Figure 7 shows the evolution of temperature, pressure, and ratio of kinetic to total energy of the system CG-12 for the first $2.5 \mathrm{~ns}$ of simulation. Other systems show similar results. Thermostats for all systems secured the convergence of temperature to our desired values and pressure response also stabilised. The proportion of kinetic energy also converged.

Figure 8 shows the configurations of systems CG-12, CG24 and CG-40 in the beginning and at the end of the simulations. The initial configurations from GCMC simulation are completely random. At the end of NVT simulations, tubes align with nearby tubes and form stacks. Shorter tubes give rise to stacks that are more distinguishable than those of longer tubes. The stacks do not exhibit observable long-range order as is found in [28], where the researchers have observed liquid crystalline columnar phase through various imaging technologies. The reason for this might be the difference between the systems in terms of tube lengths, electrolyte, $\mathrm{pH}$, and the presence of an external electric field. These could all be good orientations for further development of this research study. To

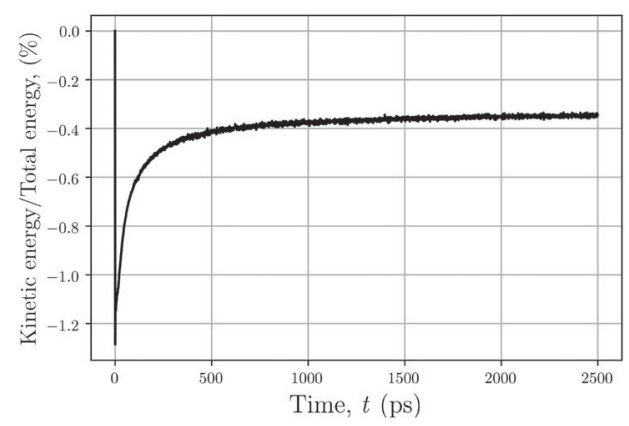

(c) energy ratio

Figure 7. Time evolution of thermodynamic variables of the system with tubes with 12 sub-particles: (a) temperature, (b) pressure, and (c) energy ratio. 


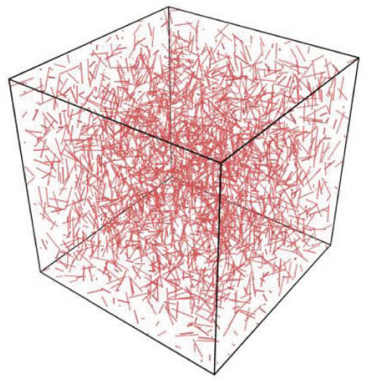

(a)

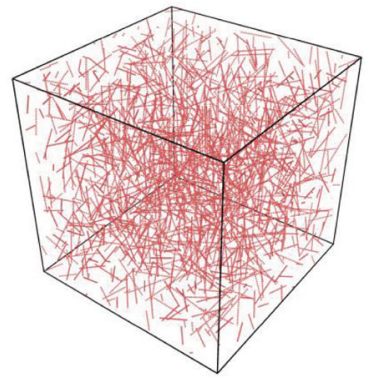

(c)

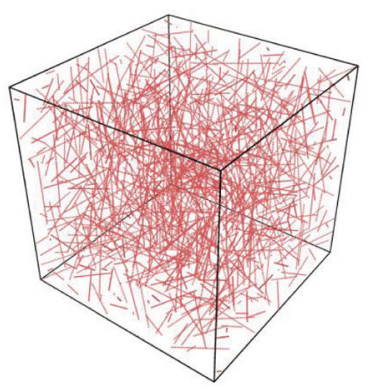

(e)

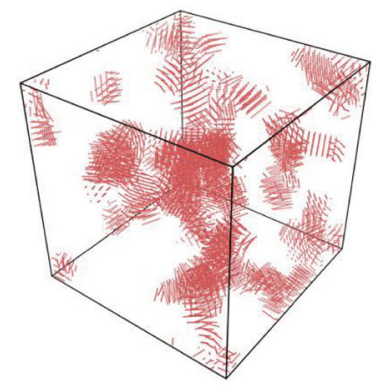

(b)

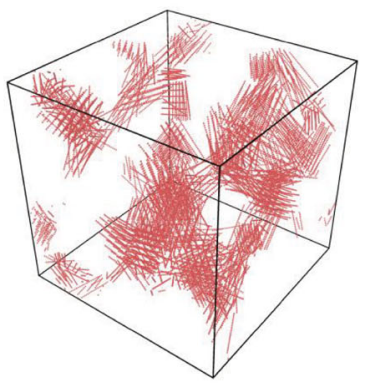

(d)

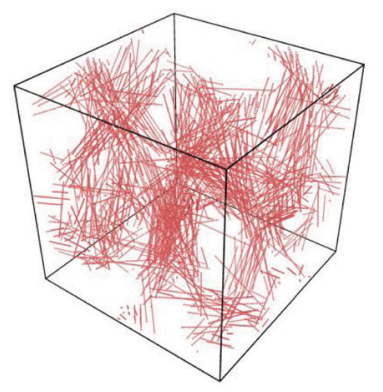

(f)
Figure 8. Configurations of three systems (CG-12, 24, and 40) in the beginning and end of the simulations. Size of the subparticle was downscaled to $3 \AA$ in radius for clearer observation of the aggregation behaviour. Rendering was achieved with OVITO [27]. (a) CG-12, initial; (b) CG-12, after 5000 ps; (c) CG-24, initial; (d) CG-24, after 6500 ps; (e) CG-40, initial; and (f) CG-40, after 8500 ps.

further analyse the geometry quantitatively, we calculated the order parameter of the assembly for snapshots taken along the process of relaxation. The order parameter $S$ was defined as in $[29,30]$,

$$
S=\left\langle\frac{3 \cos ^{2} \theta-1}{2}\right\rangle
$$

where the average was taken over all tubes and $\theta$ is the angle between the unit vector of each tube and the director vector $\mathbf{n}$ of the assembly. The director vector is defined as the major eigenvector of the order tensor,

$$
\mathbf{Q}=\frac{1}{N} \sum_{p=1}^{N}\left(\mathbf{u}_{p} \otimes \mathbf{u}_{p}-\frac{1}{3} \mathbf{1}\right)
$$

i.e. the average of the deviatoric part of the projection tensor for the unit vector $\mathbf{u}_{p}$ of tubes $p=1,2, \ldots, N$.

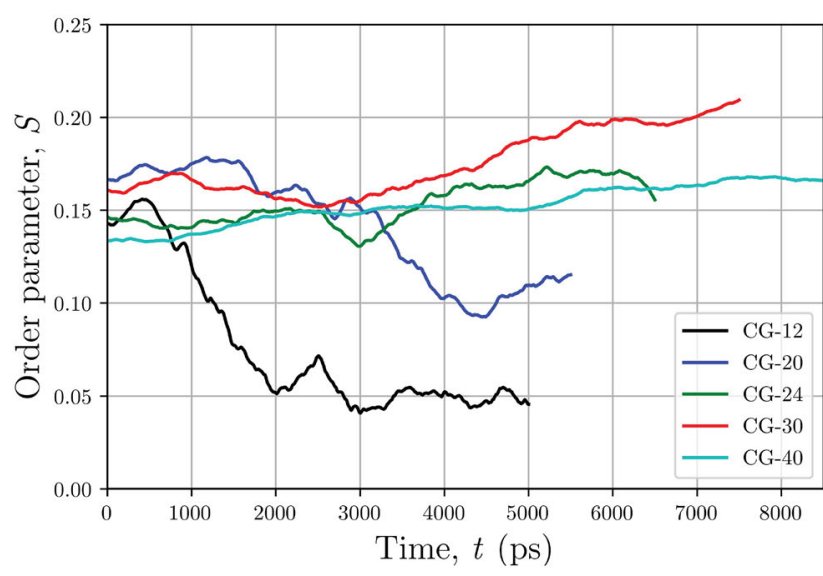

(a)

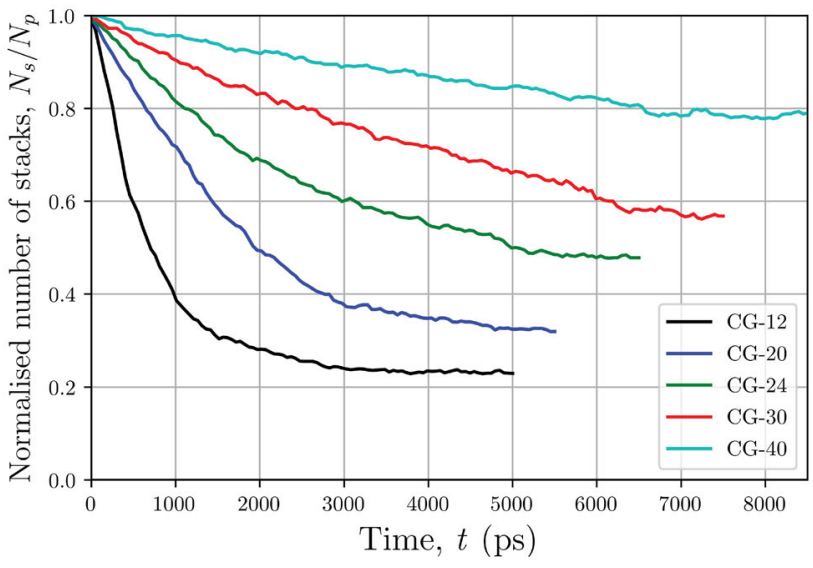

(b)

Figure 9. Evolution of order parameter (a) and normalised number of stacks (b) with time. The number of stacks are normalised by the total number of imogolite nanotubes. Individual tubes not attached to others are counted as stacks with a size of $1 . N_{s}$ refers to the number of stacks, and $N_{p}$ the total number of particles.

When the nanotubes are linearly aligned (prolate), $\theta$ will be close to 0 or $\pi$, and hence, $S \rightarrow 1$. In contrast, when $S$ is a minimum $(-0.5)$ the nanotubes are orthogonal to each other (oblate). The case with $S=0$ corresponds to random orientation (isotropic) [30].

Figure 9(a) shows the effect of tube length on the evolution of the order parameter. For all cases, $S$ remains in a small range $0.05-0.20$. This means that the initial configurations generated by the GCMC simulation are random, and the aggregation process does not give rise to a preferred orientation of the tubes, which means the orientation of the stacks formed during the process are randomly oriented. This could also be observed in Figure 10 showing the radial distribution function at the beginning and end of the simulations. In the beginning, the radial distribution functions are like those for 'gaseous phase'. This means the initial configurations are dilute and tubes are uncorrelated. At the end of the simulation, the nanotubes exhibit correlations as in the liquid phase, especially in the case of CG-20 and CG-24. 


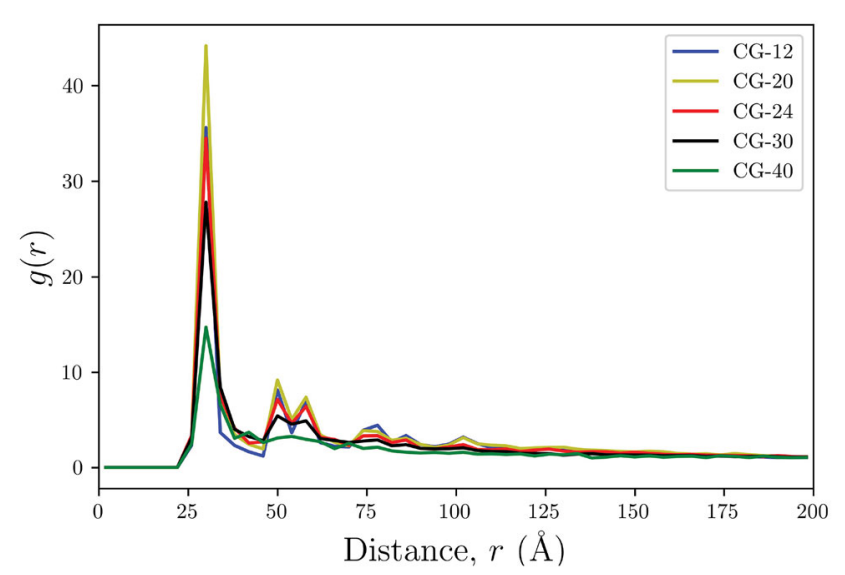

Figure 10. Radial distribution function calculated with respect to the centres-of-mass of the tubes at the end of the simulations.

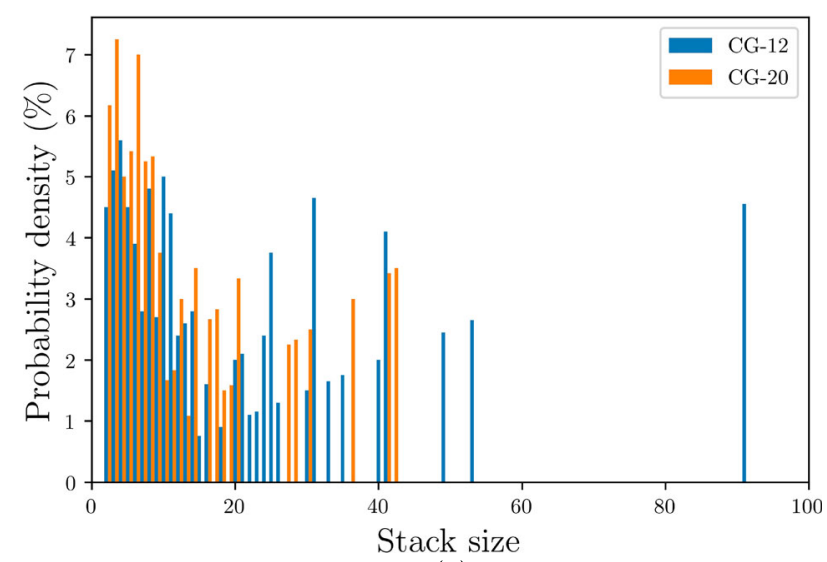

(a)

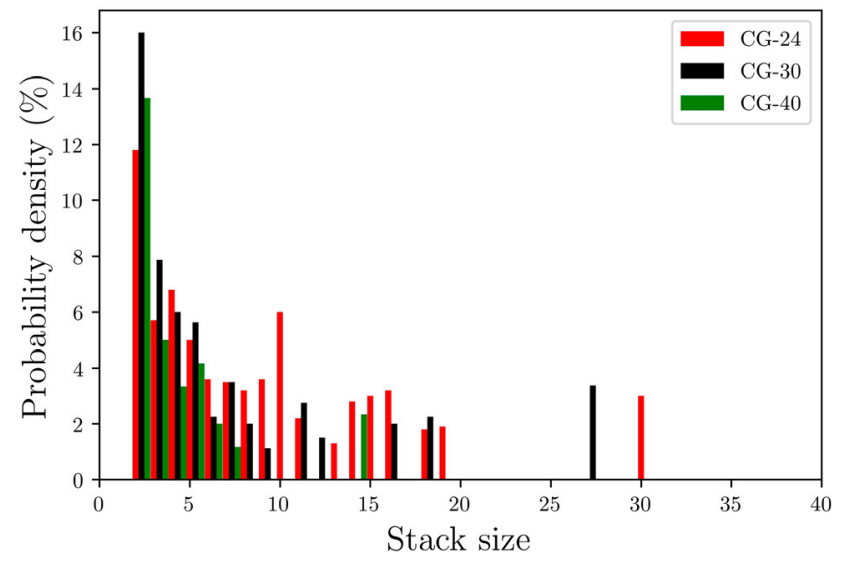

(b)

Figure 11. Probability density distribution of finding a tube in a stack of a certain size at the final configurations of systems (a) CG12 and CG-20; (b) CG-24, CG-30, and CG-40. Stacks of size 1 are not shown in the plots. (a) CG-12 and CG-20, and (b) CG-24, CG-30, and CG-40.

Apart from this, we also looked into the stack size and number of stacks in the assembly. Here a stack is defined geometrically by the following criteria:

(1) distance between centres-of-mass of the tubes is less than $40 \AA$ and angle $\theta$ between unit vectors satisfies $\cos ^{2} \theta>0.95$ (very well aligned, acute angle less than $\left.12.9^{\circ}\right)$; or

(2) if tubes A and B belong to one stack, and B and C belong to one stack, then $\mathrm{A}$ and $\mathrm{C}$ belong to one stack.

Figure 9(b) shows the evolution of the number of stacks with time. If a tube does not form any stack with other tubes, it is counted as a stack of 1 tube. The number of stacks is normalised by the total number of the tubes in the system. All systems form more stacks during the simulation process, and the number of stacks tends to level out in the end. Short tubes tend to form more stacks than longer tubes. A difference in the dimension ratio by a factor of around 3.3 (40 to 12 subparticles) can lead to a difference in the normalised stack number by a factor of around 4 (0.8 to 0.2$)$. We also study the stack size distribution of the final configurations of the systems (Figure 11). Shorter tubes like CG-12 and CG-20 have a large span of stack sizes and a relatively high proportion of the tubes form large stacks containing up to 91 tubes (CG-12). However, in the case of longer tubes (CG-24, CG-30, and CG-40), tubes tend to form less large stacks, and most tubes are found to be in stacks of 2-4 tubes.

\section{Conclusions}

In this paper, we have studied the aggregation of imogolite nanotubes with nanoscale and mesoscale MD simulations. Nanoscale simulations of two parallel tubular tubes provided us with the potential of mean force between the tubes, which was subsequently used to calibrate an effective interaction function between coarse-grained tubes. Mesoscale simulations of tubes with various lengths were carried out with the coarse-grained effective tube-tube interaction function, and their aggregation behaviour was studied. We found that shorter tubes were better at forming large aggregates or stacks of parallel tubes and present a greater potential of forming membrane-like microstructures. Further investigations are taken in the direction of altering the surface chemistry of the tubes, tuning the salinity of the solution, and adjusting the temperature and pressure of the systems to find the optimal stacking condition. We also plan to refine the representation of the computed PMF curve by either combining LJ and Yukawa potentials [31] or Fourier series [32]. We will also plan to study the mechanical properties of the tube assembly [33] as a function of their length.

\section{Acknowledgments}

This work used the Extreme Science and Engineering Discovery Environment (XSEDE) [34] Stampede2 at Texas 
Advanced Computing Center (TACC) through allocations TG-MSS170021 and TG-MSS180023, which are supported by National Science Foundation grant number ACI-1548562. Roland Pellenq and Aikaterini Ioannidou wish to thank CNRS and Aix-Marseilles Université foundation for their support.

\section{Disclosure statement}

No potential conflict of interest was reported by the authors.

\section{Funding}

The authors acknowledge the funding support from the US National Science Foundation (NSF) [ACI-1548562] under the grant no. 1702689 'Collaborative Research: Multi-scale Modelling and Measurement of Clay Aggregate Behaviour'.

\section{References}

[1] E. Shafia, S. Esposito, M. Armandi, M. Manzoli, E. Garrone, and B. Bonelli, Micropor. Mesopor. Mater. 224, 229 (2016).

[2] F. Alvarez-Ramírez, Phys. Rev. B. 76 (12), 125421-1 (2007).

[3] F. Alvarez-Ramírez, J. Comput. Theor. Nanosci. 6 (5), 1120 (2009).

[4] D. Ebrahimi, A. Whittle, and R. Pellenq, J. Chem. Phys. 140, 154309 (2014).

[5] L. Brochard, T. Honório, M. Vandamme, M. Bornet, and M. Peigney, Acta Geotech. 12, 1261 (2017).

[6] B. Carrier, Ph. D. thesis, ENPC Paris, 2013.

[7] D. Ebrahimi, R. Pellenq, and A. Whittle, Langmuir 28 (49), 16855 (2012).

[8] J. Engstler and N. Giovambattista, J. Phys. Chem. B 122 (38), 8908 (2018).

[9] S. Garcia-Garcia, S. Wold, and M. Jonsson, J. Colloid Interface Sci. 315 (2), 512 (2007).

[10] P. Cradwick, V. Farmer, J. Russell, C. Masson, K. Wada, and N. Yoshinaga, Nat. Phys. Sci. 240, 187 (1972).

[11] P. Atkins and L. Jones, Chemical Principles, the Quest for Insight (W. H. Freeman \& Co., New York, 2008).

[12] S. Plimpton, J. Comput. Phys. 117, 1 (1995).

[13] S. Nosé, J. Chem. Phys. 81, 51 (1984).

[14] W. Hoover, Phys. Rev. A. 21 (3), 1695 (1985).

[15] R. Cygan, J. Liang, and A. Kalinichev, J. Phys. Chem. B 108 (4), 1255 (2004).

[16] J. Suter, P. Coveney, H. Greenwell, and M. Thyveetil, J. Phys. Chem. C 111 (23), 174 (2007).

[17] E. Pollock and J. Glosli, Comput. Phys. Commun. 95 (2-3), 93 (1996).

[18] R. Hockney and J. Eastwook, Computer Simulation Using Particles (Hilger, Bristol, 1988).

[19] J.P. Ryckaert, G. Ciccotti, and H. Berendsen, J. Comput. Phys. 23, 327 (1977).

[20] C. Lee and H. Scott, J. Chem. Phys. 73 (9), 4591 (1980).

[21] C. Chipot and A. Pohorille, Free Energy Calculations (Springer-Verlag, Berlin Heidelberg, 2007).

[22] R. Zwanzig, J. Chem. Phys. 22, 1420 (1954).

[23] K. Heckman and C. Rasmussen, in Climate Change Impacts on Soil Processes and Ecosystem Properties, Developments in Soil Science, edited by W. R. Horwath and Y.
Kuzyakov (Elsevier, Cambridge (US), 2018), Vol. 35, pp. 93-110.

[24] J.P. Gustafsson, Clay. Clay Miner. 49 (1), 73 (2001).

[25] A. Luzar and D. Chandler, Phys. Rev. Lett. 76 (6), 928 (1996).

[26] W. Humphrey, A. Dalke, and K. Schulten, J. Mol. Graph. 14, 33 (1996).

[27] A. Stukowski, Model. Simul. Mater. Sci. 18 (1), 015012 (2009).

[28] E. Paineau, M.E.M. Krapf, M.S. Amara, N.V. Matskova, I. Dozov, S. Rouziere, A. Thill, P. Launois, and P. Davidson, Nat. Commun. 7, 10271 (2016).

[29] G. De Gennes and J. Prost, The Physics of Liquid Crystals (Clarendon Press, Oxford, 1993), pp. 41-43.

[30] D. Andrienko, J. Mol. Liq. 267, 520 (2018).

[31] K. Ioannidou, M. Kanduč, L. Li, D. Frenkel, J. Dobnikar, and E.D. Gado, Nat. Commun. 7, 12106 (2016).

[32] S. Masoumi, H. Valipour, and M.J.A. Qomi, Appl. Mater. Interfaces 9, 27338 (2017).

[33] M. Holmboe, S. Wold, and M. Jonsson, J. Contam. Hydrol. 128, 19 (2012).

[34] J. Towns, T. Cockerill, M. Dahan, I. Foster, K. Gaither, A. Grimshaw, V. Hazlewood, S. Lathrop, D. Lifka, G.D. Peterson, R. Roskies, J.R. Scott, and N. Wilkins-Diehr, Comput. Sci. Eng. 16 (5), 62 (2014).

\section{Appendices}

\section{Appendix 1. CLAYFF force field}

CLAFF is a force field proposed in [15]. The parameters was obtained using structural and spectroscopic data fitted to some simple compounds. It consists of non-bonded and bonded interactions, of which the former includes long-range Coulombic interactions and cut-off van der Waals interactions in Lennard-Jones form, and the latter includes harmonic bond and angle stretching terms, i.e.

$$
U^{\text {total }}=U^{\text {bond }}+U^{\text {angle }}+U^{\text {Coulomb }}+U^{V d W}
$$

The four terms take the following forms:

$$
\begin{aligned}
U_{i j}^{\text {bond }} & =k_{1}\left(r_{i j}-r_{0}\right)^{2} \\
U_{i j k}^{\text {angle }} & =k_{2}\left(\theta_{i j k}-\theta_{0}\right)^{2} \\
U_{i j}^{\text {Coulomb }} & =\frac{1}{4 \pi \epsilon_{0}} \frac{q_{i} q_{j}}{r_{i j}} \\
U^{V d W} & =\epsilon_{i j}\left[\left(\frac{\sigma_{i j}}{r_{i j}}\right)^{12}-2\left(\frac{\sigma_{i j}}{r_{i j}}\right)^{6}\right]
\end{aligned}
$$

where $r_{i j}, q_{i}, q_{j}$ and $\theta_{i j k}$ are the distance between atoms $i$ and $j$, their charges and the angle formed by atoms $i, j$, and $k ; k_{1}, k_{2}$, $r_{0}$, and $\theta_{0}$ are constants; $\epsilon_{0}$ is the dielectric constant of vacuum; $\sigma_{i j}$ and $\epsilon_{i j}$ are constants that are specific to atom types of atoms $i$ and $j$ and is calculated from per-type property through

$$
\begin{aligned}
\epsilon_{i j} & =\sqrt{\epsilon_{i} \epsilon_{j}} \\
\sigma_{i j} & =\frac{1}{2}\left(\sigma_{i}+\sigma_{j}\right)
\end{aligned}
$$


Table A1. Non-bonded parameters in CLAYFF force field.

\begin{tabular}{lccc}
\hline Atom type & $\begin{array}{c}\text { Charge, } q \\
(\mathrm{e})\end{array}$ & $\begin{array}{c}\epsilon \\
(\mathrm{kcal} . / \mathrm{mol})\end{array}$ & $\begin{array}{c}\sigma \\
(\AA)\end{array}$ \\
\hline Octahedral Al & 1.5750 & $1.3298 \times 10^{-6}$ & 4.7943 \\
Tetrahedral Al & 1.5750 & $1.8405 \times 10^{-6}$ & 3.7064 \\
Tetrahedral Si & 2.1000 & $1.8405 \times 10^{-6}$ & 3.7064 \\
Octahedral Mg & 1.3600 & $9.0298 \times 10^{-7}$ & 5.0909 \\
Water H & 0.4100 & 0.0 & 0.0 \\
Hydroxyl H & 0.4250 & 0.0 & 0.0 \\
Water O & -0.8200 & 0.1554 & 3.5532 \\
Hydroxyl O & -0.9500 & 0.1554 & 3.5532 \\
Hydroxyl O near substitution & -1.0808 & 0.1554 & 3.5532 \\
Clay O & -1.0500 & 0.1554 & 3.5532 \\
Clay O near octahedral substitution & -1.1808 & 0.1554 & 3.5532 \\
Clay O near tetrahedral substitution & -1.1688 & 0.1554 & 3.5532 \\
Sodium ion Na & 1.0000 & 0.1301 & 2.4178 \\
\hline
\end{tabular}

Note: In this table, 'near' means 'adjacent to'.

Table A2. Bonded parameters in CLAYFF force field.

\begin{tabular}{lccc}
\hline Type & $k$ & $r_{0}(\AA)$ & $\theta_{0}(\mathrm{deg})$ \\
\hline $\mathrm{O}-\mathrm{H}$ bond & $554.1349 \mathrm{kcal} / \AA^{2} \cdot \mathrm{mol}$ & 1.0 & - \\
$\mathrm{H}-\mathrm{O}-\mathrm{H}$ angle & $45.768 \mathrm{kcal} / \mathrm{rad}^{2} \cdot \mathrm{mol}$ & - & 109.47 \\
\hline
\end{tabular}

The parameters mentioned above are listed in Tables A1 and A2. In LAMMPS, the LJ potential takes a slightly different form,

$$
\tilde{U}_{i j}^{L J}=4 \tilde{\epsilon}_{i j}\left[\left(\frac{\tilde{\sigma}_{i j}}{r_{i j}}\right)^{12}-\left(\frac{\tilde{\sigma}_{i j}}{r_{i j}}\right)^{6}\right]
$$

Conversion between the two sets of parameters are

$$
\begin{aligned}
& \tilde{\epsilon}_{i j}=\epsilon_{i j} \\
& \tilde{\sigma}_{i j}=\frac{\sigma_{i j}}{\sqrt[6]{2}}
\end{aligned}
$$

\section{Appendix 2. Subparticle properties in mesoscale model}

The mass of a subparticle in the mesoscale simulation was obtained from longitudinal line density of the imogolite nanotube, which was $565.797 \mathrm{~g} / \AA ̊ \mathrm{~mol}$, so that

$$
M_{\text {sub }}=\lambda L=565.797 \times 8.4=4752.69 \mathrm{~g} / \mathrm{mol}
$$

The radius was calculated so as to keep the longitudinal component of the moment of inertia correct. For a spherical subparticle, $I_{\text {subparticle }}=\frac{2}{5} M R_{\text {reduced }}^{2}=I_{\text {real }}$, so that

$$
R_{\text {reduced }}=\sqrt{\frac{5 I_{\text {real }}}{2 M}}=13.635 \AA
$$

or in terms of diameter $\phi=27.27 \AA$. This is common to subparticles in all tubes. 